\title{
Head-on Immobilization of DNA Fragments on CVD-diamond Layers
}

\author{
S. Wenmackers ${ }^{1}$, P. Christiaens ${ }^{1}$, W. Deferme ${ }^{1}$, M. Daenen ${ }^{1}$, K. Haenen ${ }^{1}$, M. \\ Nesládek $^{1}$, P. Wagner ${ }^{1}$, V. Vermeeren ${ }^{2}$, L. Michiels ${ }^{2}$, M. vandeVen ${ }^{2}, \mathrm{M}$. \\ Ameloot $^{2}$, J. Wouters ${ }^{3}$, L. Naelaerts ${ }^{3}$, and Z. Mekhalif ${ }^{4}$ \\ ${ }^{1}$ Limburgs Universitair Centrum, Institute for Materials Research, Wetenschapspark 1, B-3590 \\ Diepenbeek, Belgium \\ ${ }^{2}$ Limburgs Universitair Centrum, Biomedical Research Institute, Universitaire Campus, Gebouw A, \\ B-3590 Diepenbeek, Belgium \\ ${ }^{3}$ Katholieke Hogeschool Limburg, Cel Kunststoffen, Universitaire Campus, Gebouw B, bus 3, B- \\ 3590 Diepenbeek, Belgium \\ ${ }^{4}$ FUNDP Namur, Laboratoire Interdisciplinaire de Spectroscopie Electronique, Rue de Bruxelles \\ 61, B-5000 Namur, Belgium
}

Keywords: affinity biosensors, DNA hybridisation, synthetic diamond layers, x-ray photoemission spectroscopy

\begin{abstract}
Synthetic diamond is regarded as a promising material for biosensors: it forms a stable platform for genetic assays and its biocompatibility opens the possibility for in vivo sensing. In this study the use of a thymidine linker for covalent DNA attachment was evaluated. Contact angle measurements provided a qualitative test of the initially oxidized surface. X-ray photoemission spectroscopy was used for further analysis of the oxides and for monitoring the effect of subsequent chemical treatments. The presence of FITC-labelled DNA was confirmed by confocal fluorescence microscopy. Enzyme linked immunosorbent assays indicated that this DNA was merely adsorbed on the diamond surface instead of covalently bound.
\end{abstract}

\section{Introduction}

DNA sensors are affinity biosensors based on the hybridisation of complementary DNA strands. The detection employs colorimetry or radioactive and fluorescent probes [1]. Standard substrates are poly-L-lysine coated glass, nylon, and silicon [1]. Diamond is a promising alternative platform because of its chemical inertness, durability and biocompatibility [2]. Experiments by Yang et al. [3] have demonstrated that the denaturation-rehybridisation cycle can be repeated over 30 times, implying that diamond-based DNA chips will have a superior long-term stability. Moreover, diamond can be made conductive by $\mathrm{p}$ - and n-type bulk doping, while hydrogen termination results in p-type surface conduction. These elements are prerequisites for a future generation of DNA chips with electronic read-out and direct integration with signal processing.

In 2002 Ushizawa et al. [4] have reported a procedure for the attachment of DNA to diamond powder. This method is based on thymidine - a derivative of thymine that is part of the DNA structure - as a linker for DNA to diamond. In their study diffuse reflectance infrared spectroscopy was used for characterization. Because electronic measurements would require films of diamond instead of powder, our main goal was to evaluate the effectiveness of the reported immobilization method on such films. The procedure starts with the oxidation of the diamond surface, a problem that is addressed in numerous experimental and theoretical studies [5-8]. For the different crystalline orientations present in a polycrystalline sample various oxygen-containing groups are introduced, making the situation more complicated. We used contact angle (CA) measurements, $\mathrm{x}-$ ray photoemission spectroscopy (XPS), laser scanning confocal fluorescence microscopy (LSCFM) and an enzyme linked immunosorbent assay (ELISA) to investigate the functionalisation process.

\section{Experimental}


Preparation of Diamond Substrates. The diamond samples used in our experiments were prepared by plasma enhanced chemical vapour deposition (PE-CVD). The choice of different growth parameters resulted in three different sample categories: (i) one small, single crystalline diamond sample, (ii) three freestanding films of polycrystalline diamond, referred to as "PCDx", "PCDy", and "PCDz", and (iii) two pieces of nanocrystalline diamond (NCD), referred to as "NCDx", and "NCDy". The single crystalline sample was grown with 16 standard cubic centimetres per minute (sccm) of methane gas (this is the source for carbon) and $1000 \mathrm{sccm}$ hydrogen gas. The pressure was 120 torr $(1$ torr $\approx 133 \mathrm{~Pa})$, the microwave $(\mathrm{MW})$ power $3 \mathrm{~kW}$, the substrate temperature $810^{\circ} \mathrm{C}$ and the deposition time 3 hours. This sample had a surface area of approximately $4 \mathrm{~mm}^{2}$. For the polycrystalline films different conditions were used: the amount of methane gas in comparison with hydrogen gas was varied (1 to 5\%) to optimise the smoothness of the layer. Also the pressure and MW power were chosen to obtain a substrate temperature of about $800^{\circ} \mathrm{C}$ : this promotes the growth of diamond with (100)-orientation. The surfaces were in the order of $\mathrm{cm}^{2}$ for these samples. After deposition, the polycrystalline layers were polished, which was not necessary for samples of type (i) and (iii). The nanocrystalline layers were grown for 60 minutes with $10 \mathrm{sccm}$ methane gas and $490 \mathrm{sccm}$ hydrogen gas. The pressure was 40 torr, the MW power 3 $\mathrm{kW}$ and the substrate temperature $710^{\circ} \mathrm{C}$. Of the three types of diamond samples, the properties of NCD films approach those of diamond powder the best, as NCD consists of the smallest grains and polishing of the films is unnecessary. Also, they had the largest surface, several $\mathrm{cm}^{2}$.

Surface-chemical Treatments. The immobilization was done using a thymidine linker as suggested by Ushizawa et al. [4]. Because in the first step of this procedure the introduction of carboxylic groups on the diamond is crucial, we tried different oxidation methods, referred to as "A", "B", and "C", as well as the original recipe of Ushizawa et al. [4], denoted below as "method U". Method U involves placing the diamond in sulphuric acid mixed with nitric acid first, then in $0.1 \mathrm{M}$ sodium hydroxide and finally in $0.1 \mathrm{M}$ hydrochloric acid (all chemicals supplied by Merck). Method A: in sulphuric acid with potassium nitrate (Merck) at $250^{\circ} \mathrm{C}$ for 0.5 hour, then in deionised (DI) water in an ultrasonic bath (US) and finally in fresh DI water of which the temperature is kept just below the boiling point (washing is repeated several times). Method B: in pure sulphuric acid at $250^{\circ} \mathrm{C}$ for 0.5 hour, then the same washing method as in method A. Method C: in chromic acid with sulphuric acid ('aqua regia' from RPL) at room temperature for 48 hours, and again the same washing method. In between the various oxidation methods the surface was regenerated by placing the samples in a hydrogen plasma in the CVD reactor. For this purpose, $500 \mathrm{sccm}$ hydrogen gas was used, the MW power was set to $3 \mathrm{~kW}$, and the substrate temperature was $730^{\circ} \mathrm{C}$. After the oxidation the samples were immersed in thionyl chloride (Merck) at $50^{\circ} \mathrm{C}$ during 12 hours. Subsequently, the diamond sample was placed in a solution of thymidine (Sigma Aldrich) and 4dimethylaminopyridine (Merck) in anhydrous pyridine (Merck) at $50^{\circ} \mathrm{C}$ during 12 hours. Then the surface was functionalised for the reaction with DNA.

DNA Immobilization. The DNA samples were double-stranded (ds) fragments of the phenylketonuria (PKU) gene with 250 base pairs. Polymerase chain reaction (PCR) was used to amplify this dsDNA-fragment. In the PCR process one of the strands was labelled with a fluorescent molecule fluorescein isothiocyanate (FITC) at the 5 '-end. Moreover, digoxigenin (DIG) labels, which can be detected with ELISA, were attached: 2'-deoxyuridine 5'-triphosphate (dUTP) was incorporated instead of 2'-deoxythymidine 5'-triphosphate (dTTP) and these dUTPs were labelled with DIG (PCR DIG Labelling Mix, Roche Diagnostics). To attach the PCR-DNA to the thymidine presumed to be present on the diamond, $20 \mu \mathrm{l}$ of polymerase buffer (Roche Diagnostics) with this DNA (20 ng/ $\mu \mathrm{l})$ was used, together with $1 \mu \mathrm{l} 4$ DNA ligase (isolated from E. coli, Invitrogen), $10 \mu 15 \times$ ligation-buffer and $19 \mu 1$ sterile water (Milli-Q). After 0.5 hour the surface was repeatedly (three times or more) rinsed in sterile water (Milli-Q) in order to wash off excess DNA. 
Characterization Methods. The oxidized surfaces were investigated with CA and XPS. CA gives a general impression of the hydrophilicity whereas XPS can give an indication of the chemical group in which the oxygen is present. XPS was also used for monitoring the effect of the treatments with thionyl chloride and thymidine. With confocal fluorescence microscopy and ELISA the presence of DNA was examined.

Contact Angle Measurements. The CA measurements were performed with an 'OCA 15 plus' instrument from Dataphysics with software package SCA 20. Each value, as presented in Table 1, is the average of the left and right initial contact angles determined for 2 to 8 different spots per sample (depending on the size of the sample), assuming a hyperellipsoidal shape of the drop; the measured values varied within $3^{\circ}$ around the average value.

X-ray Photoemission Spectroscopy. The XPS measurements were done on two instruments: a Hewlett-Packard 5950A ESCA spectrometer (in which only small samples can be mounted, with $0.6 \mathrm{eV}$ energy resolution, and no lateral resolution) and an SSX-100 ESCA spectrometer from Surface Science Instruments (allowing for larger surfaces, with $0.65 \mathrm{eV}$ energy resolution, and an analysis spot of $150 \mu \mathrm{m}$ ). XPS gives elemental as well as chemical information about a thin surface layer - the sampling depth in our studies was about 5 to $10 \mathrm{~nm}$. Obtained peak energies can be compared to the values in other XPS-studies on diamond and diamond like materials [9-11].

Confocal Fluorescence Microscopy. The fluorescence measurements were performed on an inverted laser-scanning confocal microscope (Zeiss LSM 510 META with software package V.3.0). The $488 \mathrm{~nm}$ line of an argon-ion laser was used for excitation, close to $495 \mathrm{~nm}$, the absorption maximum of FITC. The laser output was tuned by an acousto-optical tuneable filter (AOTF) and spectral purity was guaranteed with an extra $488 / 10 \mathrm{~nm}$ excitation interference filter. The detected emission spanned all wavelengths between $535 \mathrm{~nm}$ and $590 \mathrm{~nm}$. All $512 \times 512$ images were obtained with a $10 \times$ objective and had the size of $920 \mu \mathrm{m} \times 920 \mu \mathrm{m}$.

Enzyme linked immunosorbent Assay. The anti-DIG-antibody used was labelled with the horseradish peroxidase enzyme (HRP) (Anti-DIG-POD-Conjugate, Roche Diagnostics). The antibody was diluted 5000 times in phosphate buffered saline (PBS) (Roche Diagnostics) with the anionic detergent Tween20 (Sigma Aldrich). The diamond surface on which DNA might be present was immersed in the resulting solution with an antibody concentration of 0.03 units $/ \mathrm{ml}$. After 1 hour the unbound enzyme-labelled antibodies were removed with PBS-buffer containing Tween 20. DNA was detected by the presence of HRP. Therefore one solution is made containing hydrogen peroxide (VWR International), another containing tetramethylbenzidine (TMB) (Sigma-Aldrich). The two solutions were mixed in equal volumes and put on the diamond surface under study for 3 minutes. If HRP is present on the surface it catalyses the reduction of hydrogen peroxide and the oxidation of TMB, making the colour of the fluid blue. With a pipette this fluid was placed in a well of an ELISA plate (96-well ELISA-plate, Nunc). The reaction was stopped by adding an equal volume of $1 \mathrm{M}$ sulphuric acid (VWR International), changing the colour into yellow. In the ELISAinstrument ('Micro Plate Reader-A4 UR/R' from Eurogenetics) the absorption of $450 \mathrm{~nm}$ light (blue light) was recorded: the amount of absorption is a measure for the amount of DIG present on the surface and indirectly for the amount of dsDNA. After this measurement the diamond surface was immersed in a $2 \times$ saline sodium citrate (SSC) buffer for 15 minutes at $60^{\circ} \mathrm{C}$. Traces of this buffer were removed with PBS-buffer containing Tween 20 and the above steps are repeated starting from the hydrogen peroxide- and the TMB-solution.

\section{Results and discussion}

To study the effect of oxidation three polycrystalline diamond samples ("PCDx", "PCDy" and "PCDz") underwent the following series of treatments: regeneration in hydrogen plasma, oxidation with method A, regeneration, and oxidation with method B. For sample PCDz there followed another regeneration, and oxidation with method $\mathrm{C}$. The $\mathrm{CA}$ results can be found in Table 1 . There 
is a clear rise in the hydrophilicity of the surface after oxidation as compared to the hydrogenated situation, but there is no significant difference in CA between the different oxidation methods. This compares well to the results of Ostrovskaya et al. [7]: they reported a CA value of $93^{\circ}$ for hydrogen-terminated diamond and $32^{\circ}$ for oxidized surfaces.

\begin{tabular}{llllll} 
& PCDx & PCDy & PCDz & NCDx & NCDy \\
& CA $\left(^{\circ}\right)$ & CA $\left(^{\circ}\right)$ & CA $\left(^{\circ}\right)$ & CA $\left(^{\circ}\right)$ & CA $\left(^{\circ}\right)$ \\
\hline H-terminated & 92 & 90 & 78 & $/$ & $/$ \\
Method A & 45 & 36 & 34 & $/$ & $/$ \\
H-terminated & 91 & 90 & 89 & 91 & 97 \\
Method B & 43 & 45 & 34 & 20 & 26 \\
H-terminated & 87 & $/$ & $/$ & $/$ & $/$ \\
Method C & 36 & $/$ & $/$ & $/$ & $/$ \\
\hline
\end{tabular}

Table 1. Average contact angle for hydrogenated and oxidized surfaces of poly- and nanocrystalline diamond.

Furthermore, we investigated the result of oxidation by method $\mathrm{B}$ on the $\mathrm{CA}$ of two NCD samples: "NCDx" and "NCDy". As NCD does not need to be polished first, these films were still hydrogen-terminated due to the deposition process. CAs were determined for the fresh samples and again after oxidation with method B. As can be seen from the average CA values in the last two columns of Table 1, the same oxidation conditions resulted in a stronger hydrophilic behaviour for these samples when compared to the previous values.

To obtain more information regarding the form in which oxygen is present on hydrophilic diamond surfaces, we prepared a small sample to be mounted in the HP XPS spectrometer: the single crystal after oxidation with method A. The peak due to oxygen (Fig. 1) can be deconvoluted into four sub-peaks, representing different states of oxygen [12]. We estimated that $65.0 \%$ of all oxygen was present in the form carbonyl and hydroxyl (also as part of carboxyl). $12.0 \%$ of the peak was due to oxygen in bridge structures. The abundancy of double bonded oxygen atoms in carboxyl was estimated to be $14.5 \%$, thus enough carboxyl seems to be present to allow for the modification with chlorine. Oxygen in other forms accounts for the other $8.5 \%$. However, it should be noted that the deconvolution and the assignment of the peaks remains arbitrary to some degree.

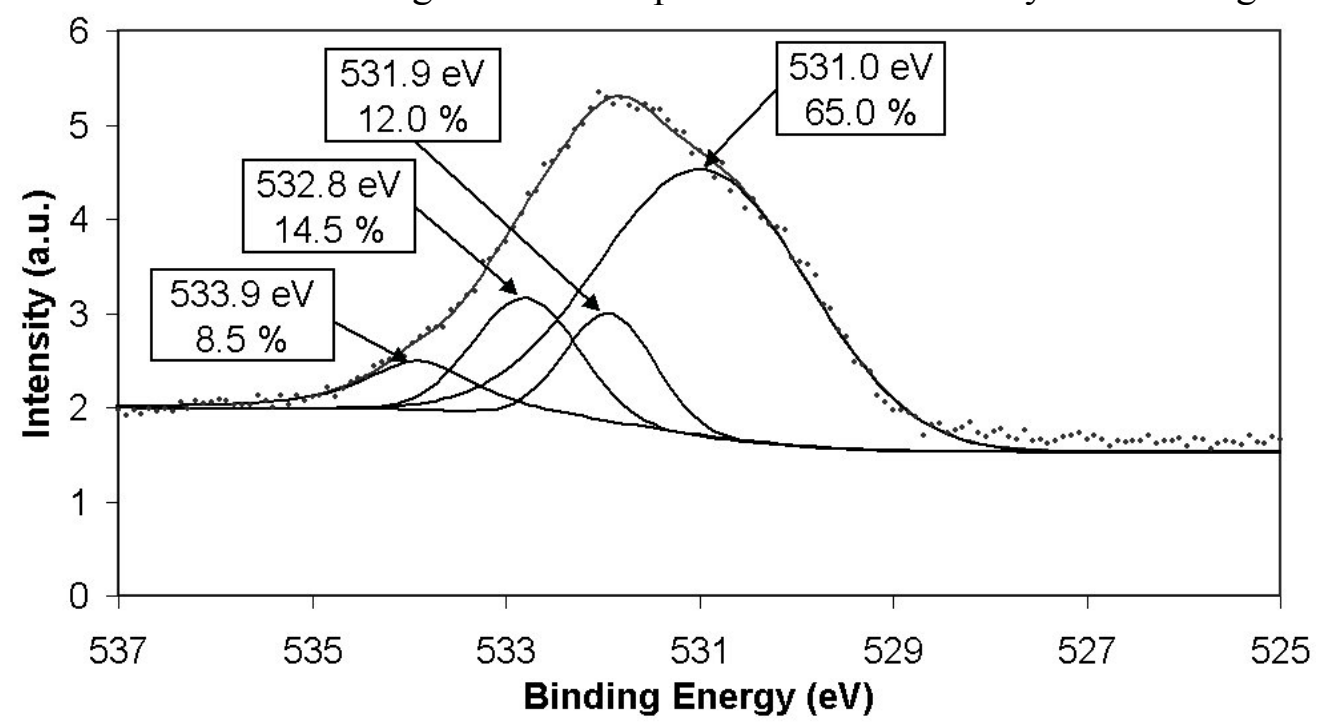

Fig. 1. Deconvolution of the oxygen 1s peak in the XPS-spectrum of a single crystal oxidized using method $\mathrm{A}$. The dots are the experimental data and the lines are the fits. 
In order to obtain chemical information of oxidized diamond, we also used XPS to obtain information on the elements present on the surface after the various chemical treatments. This was done with the SSX-100 instrument, thus the larger polycrystalline samples could be used. From the peak area in an overview scan atomic percentages can be calculated. The percentages of elements present on PCDx are given in Table 2. Similar results were obtained for other polycrystalline samples (data not shown). The twofold effect of the treatment with thionyl chloride after the oxidation is clear: chlorine, necessary for the subsequent attachment of thymidine, was introduced on the surface and the sulphur contamination was reduced. After the treatment with thymidine and pyridine less chlorine was found on the surface as expected. However, since some nitrogen was already present before the reaction with thymidine, we cannot conclude from these data alone whether also the immobilisation of thymidine (and after that of DNA) was successful. Therefore we tried to evaluate the end result by searching for the FITC-label under the microscope. After repeatedly washing of the samples with sterile water, indeed the presence of surface-bound DNA was confirmed by confocal fluorescence microscopy, see Wenmackers et al. [13]. Finally we used ELISA as an additional test for the covalent nature of the DNA-attachment.

\begin{tabular}{lcccccc}
\cline { 2 - 6 } & $\mathrm{C}(\%)$ & $\mathrm{O}(\%)$ & $\mathrm{N}(\%)$ & $\mathrm{S}(\%)$ & $\mathrm{Cl}(\%)$ & $\mathrm{Si}(\%)$ \\
\hline After oxidation method A & 60.7 & 28.8 & 4.8 & 5.8 & 0.0 & 0.0 \\
After treatment with thionyl chloride & 69.8 & 22.5 & 2.7 & 3.5 & 1.5 & 0.0 \\
After treatment with thymidine & 82.3 & 15.4 & 1.2 & 0.6 & 0.6 & 1.2 \\
\hline
\end{tabular}

Table 2. Elements present on sample PCDx after functionalisation.

If the dsDNA is present on the surface it should also be possible to detect the DIG-labels with an ELISA test. HRP-labelled anti-DIG-antibodies were allowed to bind to DIG. Then, the HRP was detected with a colour-reaction and the samples were washed with $2 \times$ SSC-buffer alternately. After a few cycles of colour-reaction and washing DIG was not detected anymore and also the fluorescence was gone. This means the dsDNA present at first and detected with fluorescence as well as (the first cycles of) ELISA, was only physically adsorbed on the surface and not covalently attached to it (since $2 \times$ SSC buffer is not able to break a covalent bond).

From the negative result of the ELISA-tests three conclusions could be drawn. Firstly it should be noted that the article of Ushizawa et al. [4] only mentions washing off the unbound DNA with water. Since we have detected DNA on our diamond samples after washing with sterile water alone, but not after washing with $2 \times \mathrm{SSC}$, their results could be explained by a shortcoming in the washing of the diamond. Indeed, it could well be that all steps resulted in the desired surface modification just as it did in our experiment according to the XPS data - except for the last step, the ligation of the DNA: DNA could be physically adsorbed on the surface, making hydrogen bonds with thymidine, but without making covalent bonds.

On the other hand it is possible that the method designed by Ushizawa et al. [4] works well for diamond powder, but it is not directly applicable to films of diamond. The observation that this method is based on carboxylic groups, which are not very abundant on our smooth oxidized surfaces, is an argument in favour of this hypothesis. The formation of carboxyl requires three dangling bonds and this is possible only for additional atoms of carbon not part of the diamond lattice. It could well be that grains of diamond show more defects, more carbon atoms with three dangling bonds, and thus more carboxylic groups after oxidation. If this is the case we should modify the first step of the procedure to obtain more carboxylic groups. We tried different oxidation methods and repeated the complete procedure on nanocrystalline diamond films, but we failed to attach dsDNA to NCD as well, making this hypothesis not very plausible. Then there is a last 
possibility: even if the amount of carboxylic groups we introduced is enough, the ligase might not be working properly close to a solid support, where it could 'wrap around' small grains.

\section{Conclusions}

As seen from contact angle measurements, the diamond surface modification by various oxidation treatments was roughly the same in all samples. In particular, after the oxidation in heated sulphuric acid and potassium nitrate, carboxylic groups account for $14.5 \%$ of the oxygen present on the diamond surface. After the further modification steps with thionyl chloride, thymidine, and FITClabelled DNA, fluorescence was detected. However after washing the surface with $2 \times$ SCC the results from both ELISA and fluorescence microscopy were negative. According to this procedure, we can not find any evidence for covalent attachment of the DNA to films of CVD diamond.

Acknowledgements This work is supported by the School for Life Sciences of the transnational University Limburg tUL, the IUAP V programme 'Quantum Size effects in Nanostructured Materials', the IWT-SBO \#030219 'CVD-diamond, a novel multifunctional material' and the Scientific Research Community WO.035.04N 'Hybrid Systems at Nanometer Scale' of FWO Flanders. KH is a postdoctoral fellow of FWO Flanders.

\section{References}

[1] S. Granjeaud, F. Bertucci, and B.R. Jordan: BioEssays 21 (1999), pp. 781-790.

[2] L. Tang, C. Tsai, W.W. Gerberich, L. Kruckeberg, and D.R. Kania: Biomaterials 16 (1995), pp. 483-488.

[3] W. Yang, O. Auciello, J.E. Butler, W. Cai, J.A. Carlisle, J.E. Gerbi, D.M. Gruen, T. Knickerbocker, T.L. Lasseter, J.L. Russell jr., L.M. Smith, and R.J. Hamers: Nature Mat. 1 (2002), pp. 253-257.

[4] K. Ushizawa, Y. Sato, T. Mitsumori, T. Machinami, T. Ueda, and T. Ando: Chem. Phys. Lett. 351 (2002), pp. 105-108.

[5] H. Notsu, I. Yagi, T. Tatsuma, D.A. Tryk, and A. Fujishima: Electrochem. Sol. Stat. Lett. 2 (1999), pp 522-524.

[6] K. Larsson, H. Björkman, and K. Hjort: Journ. Appl. Phys. 2 (2001), pp. 1026-1034.

[7] L. Ostrovskaya, V. Perevertailo, V. Ralchenko, A. Dementjev, and O. Loginova: Diamond Relat. Mater. 11 (2002), pp 845-850.

[8] P. John, N. Polwart, C.E. Troupe, and J.I.B. Wilson: Diamond Rel. Mater. 11 (2002), pp. 861866.

[9] P. Mérel, M. Tabbal, M. Chaker, S. Moisa, and J. Margot: Appl. Surf. Sci. 136 (1998), pp. 105110.

[10] S. Petrick, and C. Benndorf: Diamond Relat. Mater. 10 (2001), pp 519-525.

[11] J. Filik, P.W. May, S.R.J. Pearce, R.K. Wild, and K.R. Hallam: Diamond Relat. Mater. 12 (2003), pp. 974-978.

[12] N.W. Makau, and T.E. Derry: Surf. Rev. Lett. 10 (2003), pp. 295-301.

[13] S. Wenmackers, K. Haenen, M. Nesládek, P. Wagner, L. Michiels, M. vandeVen, and M. Ameloot: Phys. Stat. Sol. (a) 199 (2003), pp. 44-48. 